\title{
Evaluating Creep Deformation in Controlled Microstructures of Sn-3Ag-0.5Cu Solder
}

\author{
TIANHONG GU (D) ${ }^{1,2}$ CHRISTOPHER M. GOURLAY (D), ${ }^{1}$ \\ and T. BEN BRITTON (1) ${ }^{1}$ \\ 1.-Department of Materials, Imperial College London, London SW7 2AZ, UK. 2.-e-mail: \\ t.gu15@imperial.ac.uk
}

\begin{abstract}
The reliability of solder joints is affected significantly by thermomechanical properties such as creep and thermal fatigue. In this work, the creep of directionally solidified (DS) Sn-3Ag-0.5Cu wt.\% (SAC305) dog-bone samples (gauge dimension: $10 \times 2 \times 1.5 \mathrm{~mm}$ ) with a controlled $<110>$ or $<100>$ fibre texture is investigated under constant load testing (stress level: $\sim 30 \mathrm{MPa}$ ) at a range of temperatures from $20^{\circ} \mathrm{C}$ to $200^{\circ} \mathrm{C}$. Tensile testing is performed and the secondary creep strain rate and the localised strain gradient are studied by two-dimensional optical digital image correlation (2-D DIC). The dominating creep mechanisms and their temperature dependence are explored at the microstructural scale using electron backscatter diffraction (EBSD), which enables the understanding of the microstructural heterogeneity of creep mechanisms at different strain levels, temperatures and strain rates. Formation of subgrains and the development of recrystallization are observed with increasing strain levels. Differences in the deformation of $\beta$-Sn in dendrites and in the eutectic regions containing $\mathrm{Ag}_{3} \mathrm{Sn}$ and $\mathrm{Cu}_{6} \mathrm{Sn}_{5}$ are studied and related to changes in local deformation mechanisms.
\end{abstract}

Key words: $\mathrm{Pb}$-free solder, creep, microstructure evolution, recrystallization

\section{INTRODUCTION}

In the transition to lead-free electronics, ${ }^{1} \mathrm{Sn}$ 3.0Ag-0.5Cu (wt.\%) (SAC305) has become a widely used commercial solder that can outperform Sn$37 \mathrm{~Pb}$ joints in thermal cycling. ${ }^{2}$ However, the advantages of SAC305 over other compositions diminishes as the severity of the thermal cycle increase ${ }^{2}$ and SAC305 often suffers from poor drop impact reliability. ${ }^{3,4}$ Therefore, there is a need to better understand the microstructural origin of deformation and failure of SAC305 solder to develop improved solder joints.

SAC305 contains more than $95 \% \beta$-Sn with a body centred tetragonal (BCT) structure and anisotropic mechanical and thermophysical properties; e.g., the coefficient of thermal expansion (CTE) is two times

(Received April 21, 2018; accepted October 16, 2018; published online October 30, 2018) larger along the $c$-direction than along the $a$-and $b$ directions; likewise, the $c$-direction is approximately three times stiffer than the $a$ - and $b$-directions. ${ }^{5,6}$ Moreover, although the liquidus temperature of SAC305 $\left(\sim 217^{\circ} \mathrm{C}^{7}\right)$ is significantly higher than for Sn-37Pb, SAC305 has a working temperature of 60$80 \%$ of its melting temperature, which means that creep occurs even at room temperature $\left(25^{\circ} \mathrm{C}\right.$ is $\left.\frac{T}{T_{M}} \sim 0.6\right)$.

Several previous studies have examined the creep behaviour of $\mathrm{Sn}-\mathrm{Ag}-\mathrm{Cu}, \mathrm{Sn}-\mathrm{Ag}$ and $\mathrm{Sn}-\mathrm{Cu}$ solders in both bulk ${ }^{8-17}$ and joint form, ${ }^{13,14,18-29}$ where variations in stress exponent $(n)$ and activation energy $(Q)$ values are reported with different proposed mechanisms. The disagreements may be justified by differences in testing methods, temperature ranges and sample preparation methods. It is indicated that climb-controlled dislocation creep is the dominating mechanism for solder alloys because of the high dependence of secondary creep rate on the tested stresses and temperatures. ${ }^{8,14,25,28}$ By using 
transmission electron microscopy (TEM), Sidhu et al. ${ }^{13,14}$ have concluded that for several bulk and joint Sn-rich SAC alloys, the deformation is controlled by subgrain formation from $25^{\circ} \mathrm{C}$ to $130^{\circ} \mathrm{C}$. The size of the subgrain is controlled by the IMC particles $\left(\mathrm{Ag}_{3} \mathrm{Sn}\right.$ and $\left.\mathrm{Cu}_{6} \mathrm{Sn}_{5}\right)$ in the bulk. Lin et al. ${ }^{8}$ supported dislocation creep as the dominating mechanism using tensile testing of bulk Sn-3.5Ag samples. The samples underwent ductile failure and the creep resistance decreased with increasing temperature, and increased with larger IMCs volume. $^{8}$

The same mechanism was also suggested by Talebanpour et al. ${ }^{28}$ using a cylinder indenter to carry out indentation creep, by Ogawa et al. ${ }^{25}$ with indentation testing, by Chen et al. ${ }^{30}$ with in situ tensile testing and by Kariya et al. ${ }^{10}$ with tensile testing of miniature samples. The samples were deformed and showed high ductility ${ }^{8,12,23,26}$ where dislocations firstly pile up to form low-angle grain boundaries (LAGBs). As the strain rate increases, both recovery and recrystallization take place to reach an equilibrium stage. The high angle grain boundaries (HAGBs) then form with further straining, leading to void formation and crack propagation through the HAGBs during tertiary stage creep. Zhang et al. ${ }^{26,27}$ used $\mathrm{Sn}-\mathrm{Ag} / \mathrm{Cu}$ solder joints to carry out in situ creep fatigue tests. Their EBSD results reveal that the IMCs act as strain concentrators, resulting in grain subdivision in the secondary creep stage. The generation of recrystallization is caused by grain rotation with dislocation climb and grain boundary sliding. ${ }^{26} \mathrm{Xu}$ et al. $^{23}$ pre-annealed the SAC305 solder joints at $210^{\circ} \mathrm{C}$ for increasing times. Increased ductility was obtained with decrease in secondary creep strain rate from $4.7 \times 10^{-6} \mathrm{~s}^{-1}$ to $0.6 \times 10^{-6} \mathrm{~s}^{-1}$. The influence of the orientation of single crystal specimens was studied by Mukherjee et al. ${ }^{24}$ and the creep strain level decreased by 1-2 orders of magnitude when the crystal orientation along the loading direction changed from [001] to [010] and [100]. However, no microstructural evidence was provided in the reported work.

Prior research ${ }^{11,16,22,31}$ has suggested a change in mechanism at higher temperature $\left(T \geq 0.7 T_{\mathrm{M}}\right)$ to diffusion creep, i.e., a decrease in stress exponent (n). For solder composition Sn-3.9Ag-0.6Cu, Vianco et al. ${ }^{16,17}$ indicated a change in creep mechanism for bulk solder at high and low temperatures. In the low temperature regime $\left(-25-75^{\circ} \mathrm{C}\right)$, dislocations act as obstacles to reduce diffusion path, which is perhaps the result of a recovery or recrystallization assisted diffusion mechanism, while the high temperature regime $\left(75-160^{\circ} \mathrm{C}\right)$ predominantly occurred by lattice or bulk diffusion mechanisms. This change in mechanism is supported by Lee et al. ${ }^{22}$ using $\mathrm{Cu} / \mathrm{Sn} / \mathrm{Cu}$ single-lap specimens, Mayer et al. $^{31}$ who tested both pure $\mathrm{Sn}$ and $\mathrm{Sn}-\mathrm{Ag}_{3} \mathrm{Sn}$ eutectic with micropillar compression, and Mathew et al. ${ }^{11}$ showed good agreement between solder- bump array samples under shear loading and bulk samples through tensile testing at room temperature.

Similar microstructural deformation mechanisms were suggested in terms of different testing methods, such as shear testing, ${ }^{32,33}$ thermal cycling, ${ }^{6,34}$ thermal shock ${ }^{35,36}$ and thermomechanical fatigue $^{5,37}$ for solder joints. They concluded that the tested solder joints deformed by continuous subgrain rotation, which controls the recrystallization of the grains. The subgrains were formed at locations with IMCs which concentrates the dislocations.

As we know, the SAC solder joints form a microstructure of $\beta$-Sn dendrites and $\beta$-Sn + intermetallic compound (IMC) eutectic regions after solidification, which contains $\mathrm{Ag}_{3} \mathrm{Sn}$ and $\mathrm{Cu}_{6} \mathrm{Sn}_{5}$ eutectic IMCs. ${ }^{38,39}$ Also, each solder joint has different $\beta$-Sn crystal orientations and number of grains. ${ }^{39-42}$ For SAC305, each joint normally has $1-3 \beta$-Sn grains, typically as a single grain joint or the joint contains cyclic twinned grains. ${ }^{43-46}$

In the present work, directional solidification (DS) by Bridgman growth is applied to generate SAC305 with $1-3$ reproducible $\beta$-Sn orientations $([110]+[100]$ along the growth direction) and eutectic $\mathrm{Ag}_{3} \mathrm{Sn}$ and $\mathrm{Cu}_{6} \mathrm{Sn}_{5}$ IMCs with reproducible size and spacing, ${ }^{47,48}$ to generate controlled microstructures relevant to SAC305 solder joints. ${ }^{39,40,42,43}$ The creep behaviour of these controlled microstructures is then investigated under constant load testing at a range of temperatures $\left(20-200^{\circ} \mathrm{C}\right)$. The secondary creep strain rate, the dominating creep mechanism, and its temperature dependence are explored. The microstructural evolution mechanisms of the sample are examined by electron backscattered diffraction (EBSD) and are mainly focused on the onset of secondary and tertiary creep. The strain field of the sample is measured by two-dimensional digital image correlation (2-D DIC). The role of IMCs in the eutectic within the bulk matrix, the variation of crystal orientations and the number of grains, i.e., the presence of a grain boundary, of each sample are considered during creep testing to investigate their effect on strain accumulation and microstructural evolution on creep reliability of the SAC305 solder alloys. These observations could be extended for understanding thermal loading of solder joints.

\section{EXPERIMENTAL PROCEDURE}

A dog-bone shape was designed to confine creep deformation to the narrow-gauge region. The samples were initially cut from a flat cold-rolled SAC305 sheet (which was rolled from a SAC305 metal bar to $1.5 \mathrm{~mm}$ thickness). The dog-bone shape was cut using electrical discharge machining (EDM) into a 10 (gauge length) $\times 2 \times 1.5 \mathrm{~mm}$ dimension (Fig. 1a). The rolled and machined sample has multiple and small grains with random orientations (Fig. 1c). In this experiment, controlled microstructures were 
(a)

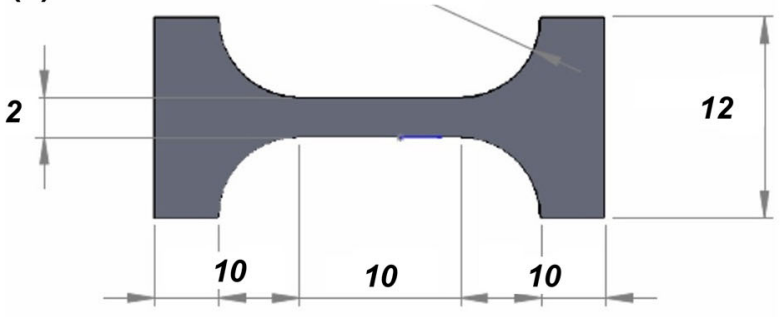

(c)
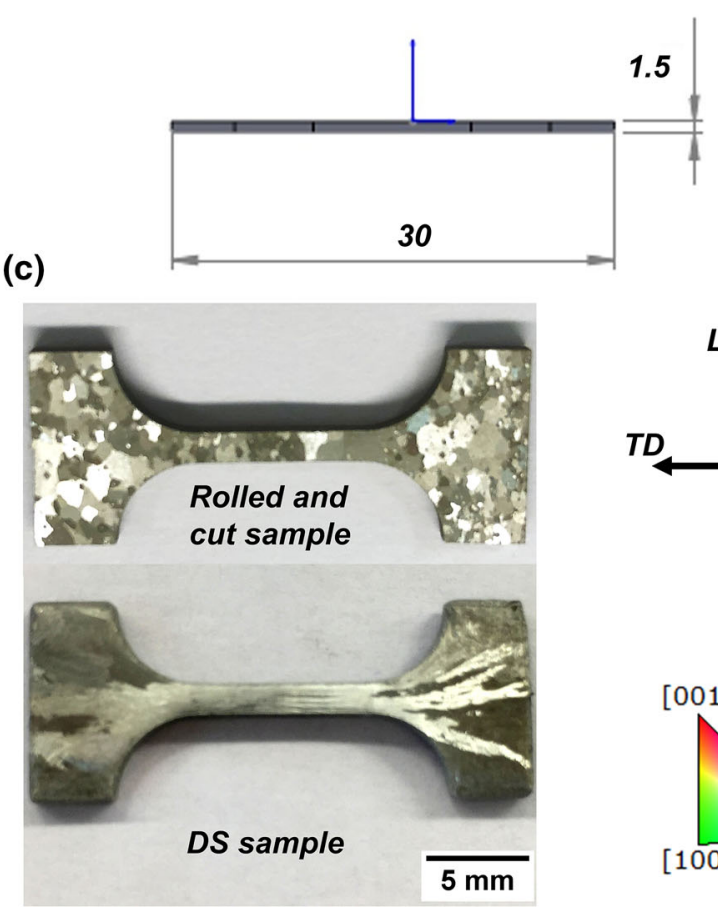

(b)

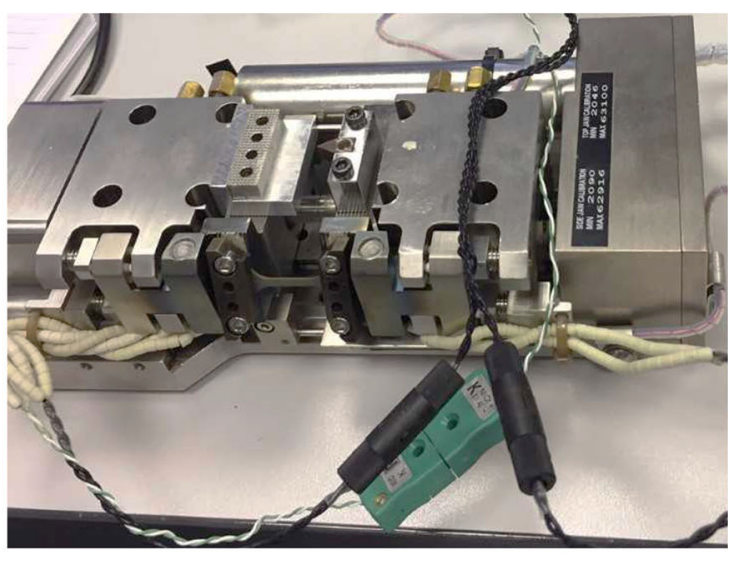

(d)

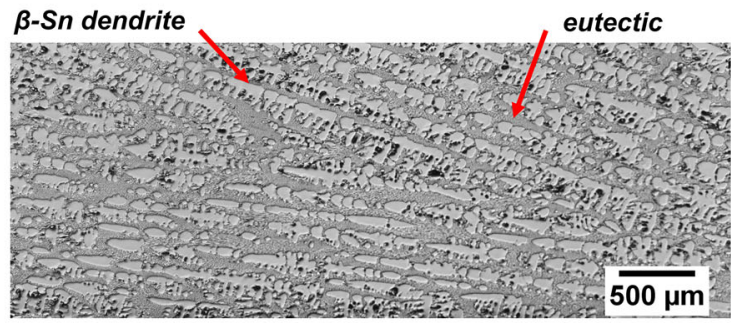

(e)

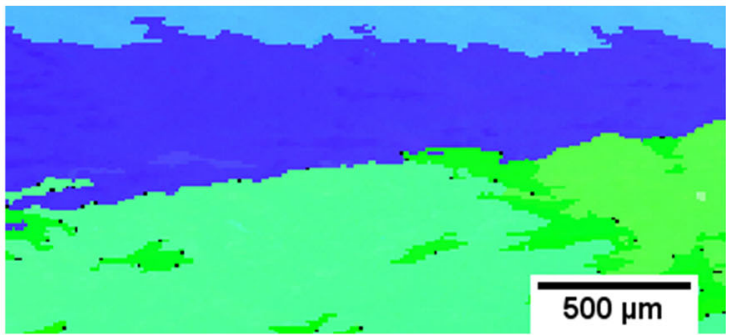

Fig. 1. (a) Schematic diagram of the SAC305 dog-bone samples for creep testing with dimensions in mm labelled. (b) The Gatan Mtest $2000 \mathrm{E}$ tensile stage with a $2 \mathrm{kN}$ load frame. (c) Optical image of the rolled and as directionally solidified (DS) samples after $\beta$-Sn etching. Note that only DS samples were used for creep testing. (d) Optical image of the DS sample with $\beta$-Sn dendrite and eutectic labelled. (e) Crystal orientations represented with inverse pole figure colouring with respect to the loading direction (IPF-LD) of the DS sample.

grown in dog-bone samples by Bridgman growth in a temperature gradient furnace with a $350^{\circ} \mathrm{C}$ hot zone and $\sim 25^{\circ} \mathrm{C}$ cold zone, similar to that used in Ref. 49. Subsequently, the rolled sample was directionally solidified (DS) by pulling the dog-bone along its length at a constant velocity of $20 \mu \mathrm{m} / \mathrm{s}$ through a temperature gradient of $\sim 3 \mathrm{~K} / \mathrm{mm}$. This produced dog-bone samples with a structure of $\beta$-Sn dendrites and interdendritic eutectic containing $\beta$-Sn, $\mathrm{Ag}_{3} \mathrm{Sn}$ and $\mathrm{Cu}_{6} \mathrm{Sn}_{5}$ (Fig. 1d), with 1-3 long columnar $\beta$-Sn grains along its gauge with a controlled orientation between $<100>$ and $<110>$ in its loading direction (LD) (Fig. 1e). These DS samples were used for mechanical testing.

The sample was then fitted inside the $2 \mathrm{kN}$ Gatan Mtest2000E tensile stage for creep testing (Fig. 1b). Load controlled deformation was performed using closed loop control within the Microtest stage, and the tests were carried out at a constant applied stress of $30 \pm 2 \mathrm{MPa}$. Deformation was performed at a range of temperatures, including room temperature, $45^{\circ} \mathrm{C}, 60^{\circ} \mathrm{C}, 90^{\circ} \mathrm{C}, 120^{\circ} \mathrm{C}, 150^{\circ} \mathrm{C}, 180^{\circ} \mathrm{C}$ and $200( \pm 0.4)^{\circ} \mathrm{C}$. The tests were interrupted shortly after the onset of secondary creep (after $\sim 120 \mathrm{~s}$ ) and stopped at the end of tertiary creep stages. The sample was removed from the load frame and EBSD scans were performed in the SEM at these two stages. The sample was then replaced within the loading frame to resume the creep test between the two EBSD scans. The EBSD maps were scanned using a Bruker e-Flash ${ }^{\mathrm{HR}}$ EBSD detector inside a Quanta FEG 450 SEM with a step size of $6 \mu \mathrm{m}$ and $0.4 \mu \mathrm{m}$ for large scale and high magnification inverse pole figure (IPF) maps, respectively. The data was analysed by Bruker ESPRIT 2.1 software during scanning and the EBSD maps were postprocessed with "absorb surrounded zero solutions".

Samples were prepared to a high-quality surface finish suitable for both DIC and EBSD. This was achieved using mechanical polishing with a $0.05 \mu \mathrm{m}$ 
colloidal silica finish. Due to the fact that the $\beta$-Sn is much softer than the eutectic IMCs, slight roughness was generated between the $\beta$-Sn and IMCs during mechanical polishing. Therefore, the final specimen surface was polished with broad ion beam milling using a Gatan PECS II to firstly flatten the specimen surface and then was introduced a very slight surface roughness which is suitable for DIC. The PECS II was operated in etching mode at a beam voltage of $3 \mathrm{keV}$ and a tilt angle of $1^{\circ}$ to flatten the IMCs, followed by $3 \mathrm{keV}$ and $3^{\circ}$, in order to generate long strip speckle patterns on the surface. Ion polishing was performed for $3 \mathrm{~h}$ in total.

Creep strain curves were measured by 2 -D optical DIC. The curves were formed from fields of the normal strain along the loading direction, extracted from the surface strain field plots by taking the arithmetic mean of each strain field map at each time-step. DIC was performed within DaVis with the following DIC settings: window size 65 pixels, overlap 16 pixels, field of view $10 \times 2 \mathrm{~mm}$, i.e., $\sim 860 \times 174$ pixels, with images captured at 14 bit depth, and an effective pixel size of $11.5 \mu \mathrm{m} /$ pixel. Each optical micrograph was captured with a QImaging Retiga QIClick ${ }^{\mathrm{TM}}$ camera attached to a Brunel Microzoom inspection microscope operating at a frame rate of $1 \mathrm{~Hz}$.

\section{RESULTS AND DISCUSSION}

\section{Creep Strain Curves}

The creep strain curves for primary creep and the early stages of secondary creep (the first $120 \mathrm{~s}$ ) are given in Fig. 2a for tests at 298-473 K. From the creep strain rate versus strain curves in Fig. $2 \mathrm{~b}$, a linear relationship with a constant gradient close to 0 is presented once secondary creep is achieved (Fig. 2b), which allows the onset of secondary creep to be identified.

The strain at the onset of secondary creep decreases with the testing temperature (Fig. 2c), especially between $298 \mathrm{~K}$ and $333 \mathrm{~K}$. This might be caused by increasing dislocation climb at higher temperature (298-333 K), which enables recovery to more easily balance the work hardening. At even higher temperature (363-473 K), the deformation mechanism changes gradually with an inflection at $\sim 343 \mathrm{~K}$, as indicated by Fig. $2 \mathrm{~d}$. This transformation could be a change from dislocation limited processes to diffusion limited deformation processes. Towards the melting temperature $\left(T_{\mathrm{M}}=490\right.$ $\mathrm{K})$ the onset of secondary strain rate tends towards zero. It is anticipated that onset of secondary creep will occur at even greater strains at lower temperatures, as there is a total reduction in creep deformation at these lower temperatures due to the thermal activation of dislocation motion.

Figure $2 \mathrm{~d}$ shows a plot of ln (secondary creep strain rate) versus the reciprocal temperature. Analysis of the gradient of this data enables calculation of the activation energy, $Q$. The data can be represented by two different temperature ranges with separate linear fits. From room temperature to $333 \mathrm{~K}$ the $Q$ is $41 \mathrm{~kJ} / \mathrm{mol}$, implying that a climbcontrolled dislocation mechanism is the predominant mechanism in this low temperature regime. A much flatter slope occurs beyond $363 \mathrm{~K}$ with a $Q$ value of $6 \mathrm{~kJ} / \mathrm{mol}$, suggesting a fast diffusion path might take place, probably due to the change into a lattice diffusion mechanism assisted by recovery and recrystallization in the high temperature regime. This is also supported through observation of a decrease in the strain at onset of secondary creep at higher temperatures (Fig. 2c), i.e., the recovery process happens at lower creep strain level because of less dislocation formation and motion, but more diffusion occurs. This change in creep mechanism happens at $\frac{T}{T_{M}} \sim 0.7, \sim 343 \mathrm{~K}$, which is in agreement with some literature ${ }^{16,17,22}$ that the change in mechanism from climb-controlled dislocation creep to lattice diffusion creep takes place at $70-80 \%$ of the melting temperature depending upon the initial microstructure of samples.

The creep curves from the onset of secondary creep until a creep strain of $\sim 10 \%$ are shown in Fig. 2e (obtained by 2-D DIC). It can be seen that, overall, a shorter secondary creep time (and strain, Fig. $2 b$ ) was obtained with increasing temperature. The creep strain magnitude at failure is attributable to the temperature and also the initial crystal orientation and number of $\beta$-Sn grains in the sample. Supplementary Table S1 summarises the creep data and the orientation and number of $\beta$-Sn grains in each sample. The secondary creep strain rates increase with increasing temperature from the order of $10^{-4} \% \mathrm{~s}^{-1}$ to $10^{-3} \% \mathrm{~s}^{-1}$. The samples containing an initial near [110] crystal orientation achieve a relatively low creep strain at the end of testing, $6.8 \%$ and $5.5 \%$ for 298 and $318 \mathrm{~K}$ (low temperature regime: $298-333 \mathrm{~K}$ ), and $26.2 \%$ and $34.2 \%$ for 453 and $473 \mathrm{~K}$ (high temperature regime: 363-473 K), whilst the samples with main orientations near [100] achieve a higher creep strain $16.9 \%$ and $42.3 \%$ at fracture for the lower and higher temperature regime, respectively. (The sample tested at $393 \mathrm{~K}$ and $473 \mathrm{~K}$ was not fractured). In tension, a total creep strain before failure can be larger along the [100] crystal direction, than [110]. The process of microstructural change is explored by EBSD in the next section.

\section{Strain Field and Crystal Orientation of [100] [110] Bi-crystal Sample}

The large scale IPF map in Fig. 3a shows the initial crystal orientations and grain structure of the bi-crystal sample tested at $120^{\circ} \mathrm{C}\left(\frac{T}{T_{M}} \sim 0.8\right)$. There are two major crystal orientations and these two orientations are near [110] and [100] in the loading direction (LD).

The DIC figures show that the bi-crystal sample has significant deformation heterogeneity in this 


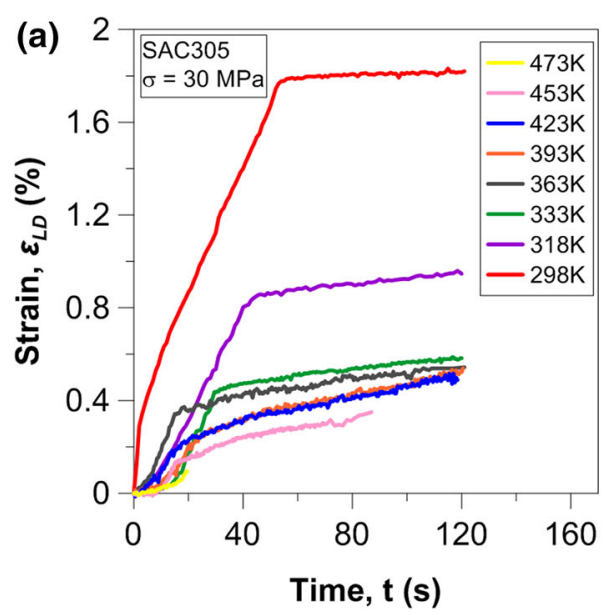

(c)

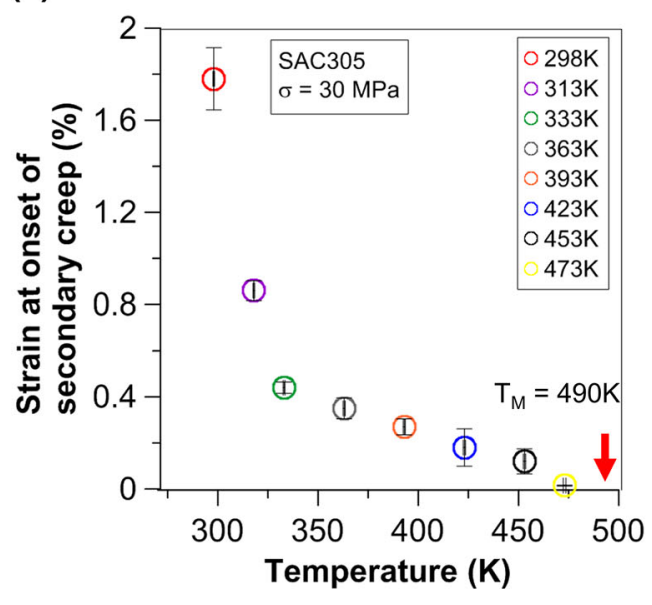

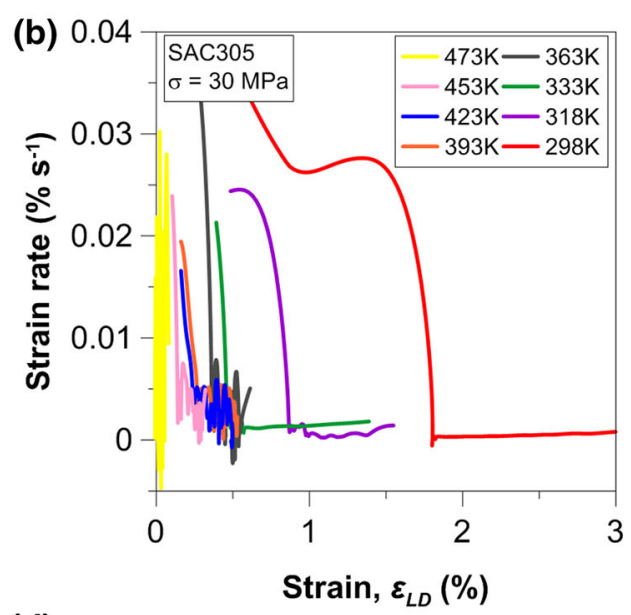

(d)

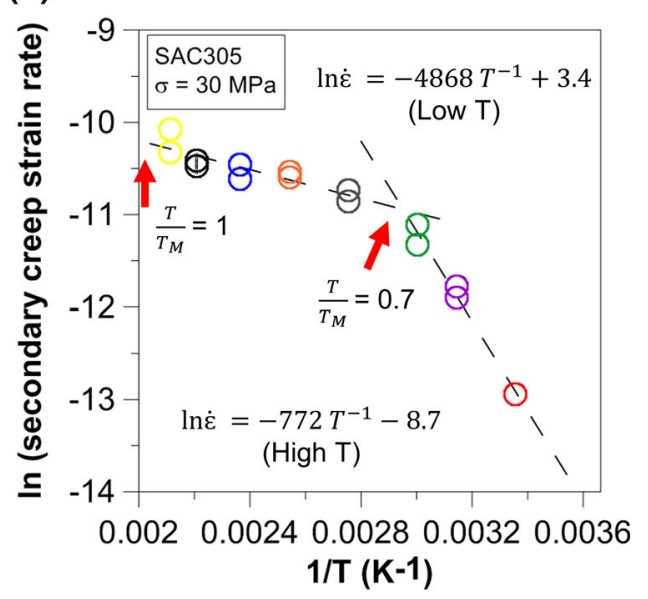

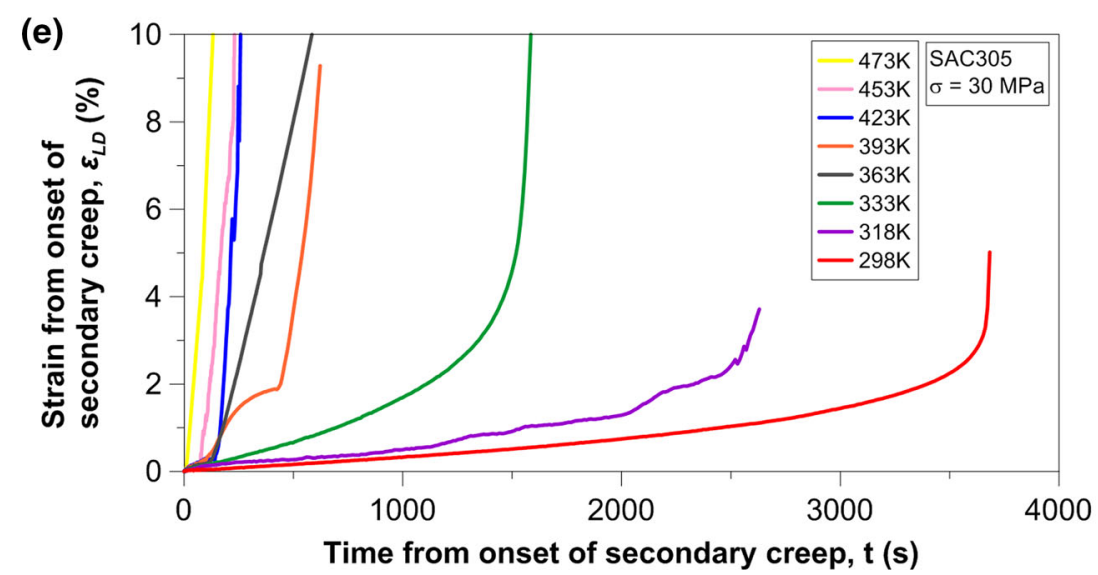

Fig. 2. Mechanical creep data from fixed load testing of SAC305 samples from $298 \mathrm{~K}$ to $473 \mathrm{~K}$. (a) Creep curves from $298 \mathrm{~K}$ to $473 \mathrm{~K}$ at primary and early secondary stages creep. (b) Secondary strain rate versus creep strain curves with a gradient of 0 shown after the secondary stage creep is reached. (c) Variation of the onset of secondary creep with respect to temperature. (d) Creep model analysis of In(secondary creep strain rate) versus reciprocal temperature with two creep domains (high temperature and low temperature). (e) Creep curves from the onset of secondary to tertiary stage creep (maximum strain $=10 \%$ ). (The symbols are included for completeness and represent orientations, as described in Supplementary Table S1).

length scale during creep straining. The high strain field starts to develop within one particular section highlighted by the red square in Fig. $3 \mathrm{~b}$. The sample has a mean strain of $0.54 \%$ at the onset of secondary stage creep (Fig. 3b and c). Most of the creep strain initiated at the interface between the blue [110] and the green [100] crystal orientations on the IPF-LD map in Fig. 3a, and the [110] grain seems to accumulate more creep strain at this stress level.

The frequency histogram (Fig. 3h) illustrates the spatial variations in strain field between Location 1 and 2 near the onset of secondary creep at $120 \mathrm{~s}$ 


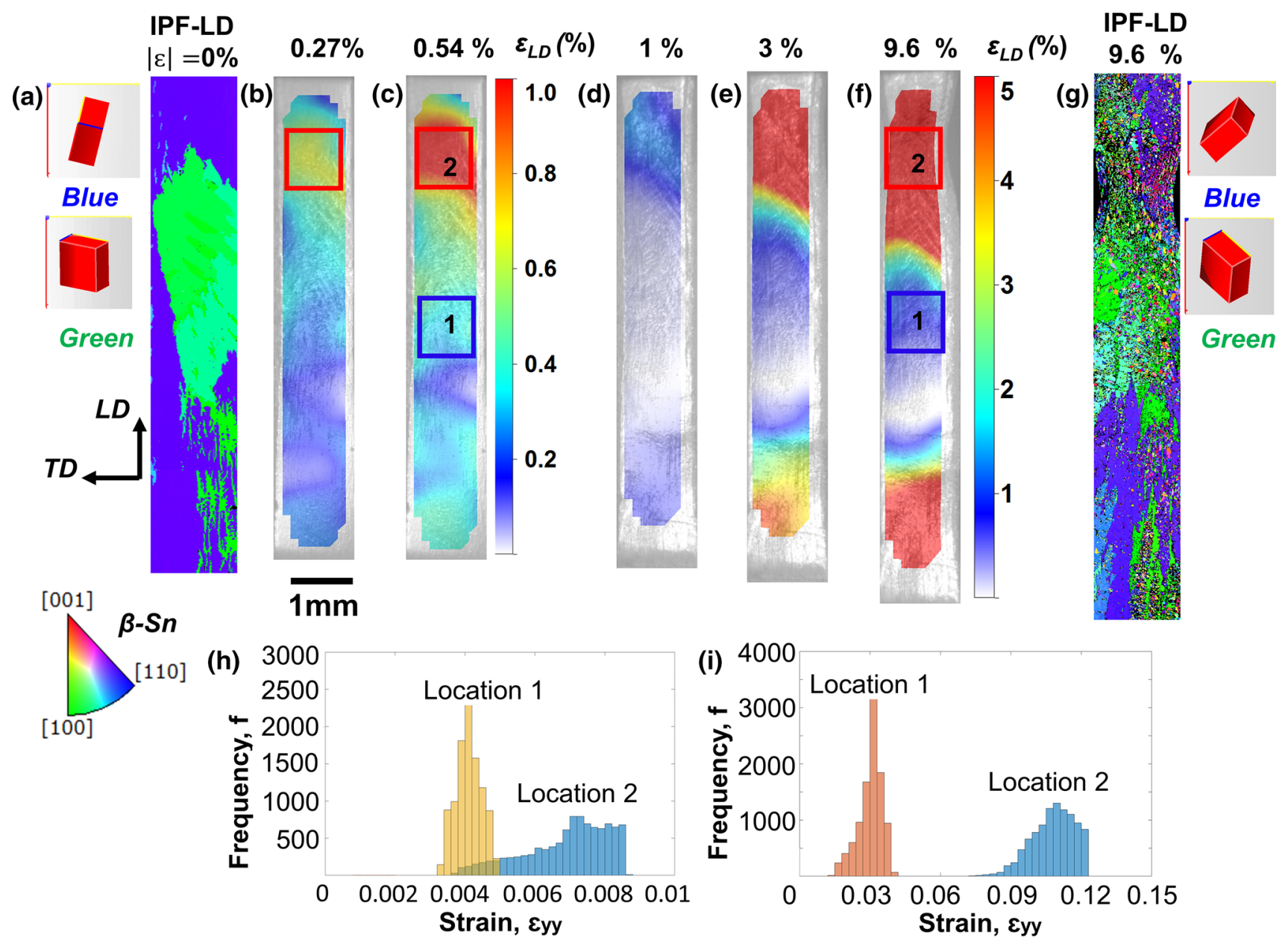

(j)
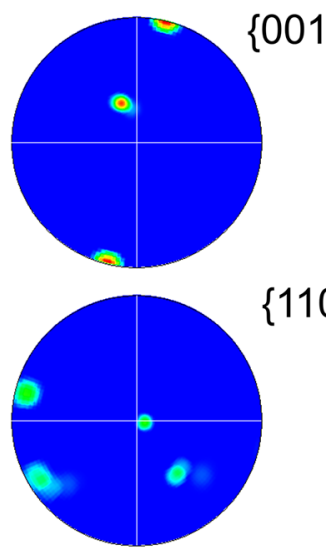

$\{100\}$

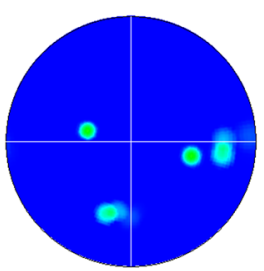

$\{110\}$

DS (k)
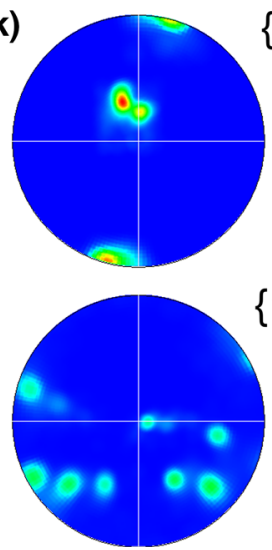

$\{001\}$

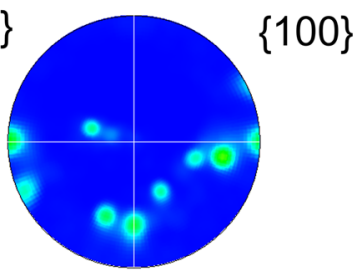

$\{110\}$

Tertiary stage

Fig. 3. The DIC figures and IPF-LD maps of a bi-crystal sample tested at $120^{\circ} \mathrm{C}$. (a) The IPF-LD map after DS $\left(\varepsilon_{\mathrm{LD}}=0 \%\right)$ with crystal orientations shown. (b, c) DIC measurements after interruption near the onset of secondary stage creep at strain $\left(\varepsilon_{\mathrm{LD}}\right)$ level $0.27 \%$ and $0.54 \%$, respectively. (d-f) DIC figures from secondary to tertiary stage creep at strain level $1 \%, 3 \%$ and $9.6 \%$ respectively. (g) The IPF-LD map at tertiary stage creep $\left(\varepsilon_{\mathrm{LD}}=9.6 \%\right)$ with the crystal unit cell of the main orientations shown. (h, i) Frequency versus local strain histogram at locations 1 and 2 shown at onset secondary stage creep (h), and at tertiary stage creep (i). (j, k) The pole figure (PF) of the sample at DS condition (j), at tertiary stage (k). (Videos of the DIC fields are found in the Supplementary video S1 and S2).

(extracted from Fig. 3c). Location 2 generates $0.8 \%$ of strain and has a main blue orientation close to [110], while Location 1 has about $0.4 \%$ of strain with a main green crystal orientation near [100], which is half of the location 2 .
As the testing continues, more strain is accommodated at the two ends (Fig. $3 \mathrm{~d}-\mathrm{f}$ ). A rapid increase in strain rate is obtained when the sample entered the tertiary stage creep. The sample forms a semi-stable neck which initiates near the grain 
boundary at Location 2, which is localising the heterogeneous deformation especially in the grain boundary region (Fig. 3f). During necking, there is significant localisation of creep strain (Fig. 3f) and surface roughening. The EBSD map (Fig. 3g) indicates that there is significant grain fragmentation and a new grain orientation is observed in this region (likely due to recrystallization).

Figure 3i gives the frequency histogram at Location 1 and 2 at the end of the creep test (extracted from Fig. 3f). The localised strain at Location 2 is $\sim 12 \%$ on average, while Location 1 only has $\sim 2.5 \%$ strain, which means that the heterogeneity of deformation increases during creep. The orientation spreading and formation of new orientations are observed by comparing the pole figure (PF) map in the DS-ed condition (Fig. 3j) with the one in tertiary stage creep (Fig. 3k), which indicates the gradual rotation of orientation and generation of recrystallization. The details of the microstructure within the two grains are explored in the next section.

\section{Microstructure of the [100] [110] Bi-crystal Sample Within the Non-necking Region}

The microstructure of the bi-crystal sample within the non-necking region, i.e., location 1 in Fig. $3 c$ and $f$, is given at different stages of creep in Fig. 4b-g, which come from the red squared region in Fig. 4a. As shown in Fig. 4a, there are four noticeable grain orientations in this location after DS. Two small grains (labelled as 1 and 2) are embedded inside the main orientation, which is close to [100].

The subgrains are formed quite early as shown in Fig. 4b and c. Their growth is preceded by the formation of significant misorientation (as indicated with higher misorientation to average, MO av., plots) and these are localised around IMCs and annotated with red arrows in Fig. 4c, even in the DS condition. Regions of high misorientation area are also generated within the dendrite, which are annotated with yellow arrows in Fig. 4c, likely due to CTE mismatch between the $\beta$-Sn dendrite and IMCs and the softness of $\beta$-Sn. Overall, the initial deformation is heavily length scale modulated by the IMCs.

For deformation in the early stages of secondary creep, the MO av. decreases smoothly across the map as given in Fig. 4e with red and yellow arrows labelled for eutectic and $\beta$-Sn dendrite regions, respectively. The two small grains 1 and 2 are removed from the initial microstructure (Fig. 4d), which might be caused by recovery processes where the material is annealing because deformation happens at $\frac{T}{T_{M}} \sim 0.8\left(T_{\mathrm{M}}\right.$ of $\left.\mathrm{SAC} 305 \sim 490 \mathrm{~K}\right)$ in this test. At this high temperature $\left(\frac{T}{T_{\mathrm{M}}} \sim 0.8\right)$, the subgrain formation becomes stronger and faster, because there is a greater amount of dislocation climb and grain boundary mobility. This might be a result of initiation of bulk diffusion mechanism as well since exceeding $\frac{T}{T_{\mathrm{M}}} \sim 0.7$, the creep mechanism changed in Fig. 2 d.

Figure $4 \mathrm{f}$ and $\mathrm{g}$ illustrate that the high misorientation regions developed more significantly at the tertiary stage creep with a creep strain level (average in location 1) about 2.5\% (Fig. 3f). These regions are introduced in the eutectic region as a result of the heterogeneity associated with the presence of IMCs with red arrows labelled. It is noticed that a larger high misorientation region (Fig. $4 \mathrm{~g}$ ) is developed in the same region where the two small grains 1 and 2 were located initially and spreads towards the initial grain boundary on the lower right of the map. Perhaps, the grain boundary is concentrating the dislocations and causing the formation of the subgrain. Although recrystallization has not been retriggered here yet, the start of the recrystallization story can be seen visibly. It is believed that this particular region with main orientation near [100] grain has not reached tertiary stage creep at the end of the test.

\section{Microstructure of the [100] [110] Bi-crystal Sample Within the Necking Region}

In the necking region shown in Fig. 5, the sample has been locally strained to about $12 \%$ (as measured from DIC, in Fig. 3f). The surface roughness of the sample (Fig. 5b) increased significantly in this region compared to the non-necked region (Fig. 4). Recrystallization was generated as illustrated in Fig. 5a, and the recrystallized grains (Fig. 5c and e) have a similar length scale as the formed subgrains in the early stage (Fig. $4 \mathrm{f}$ and g). These recrystallized grains tend to have minimal internal misorientation (as expected from a recrystallization process) and the misorientation maps (Fig. 5d and f) show that some recrystallized grains are close in orientation. Locations within the sample have high MO av., which increases greatly to $15^{\circ}$, as given by the Fig. 5d with $\mathrm{MO}$ av. per grain map colour key 0 $15^{\circ}$. A high MO av. implies significant local constraint which results in an increase in crystal rotation. ${ }^{50}$

The centre of the necking region (Fig. 5e and f) has a greater amount of local deformation in this region. Figure $5 \mathrm{f}$ shows that the constrained misorientation (Fig. 5d) has been released due to the generation of recrystallization. It can be seen that the MO av. is still relatively higher in the eutectic region around IMCs than around the $\beta$-Sn dendrites. It is observed from Fig. 5e that the $\beta$-Sn within the eutectic region has similar 'rainbow colour' recrystallization as the $\beta$-Sn within the dendrite, but the $\beta$-Sn in the eutectic has a much smaller grain size. It is believed that the deformation of the sample is initiated in the $\beta$-Sn within the eutectic region near IMCs and runs through the $\beta$ Sn dendrite as evidenced by in-grain lattice rotations. An increase in the in-grain lattice rotations corresponds to an increase in stored energy, and 
(a)
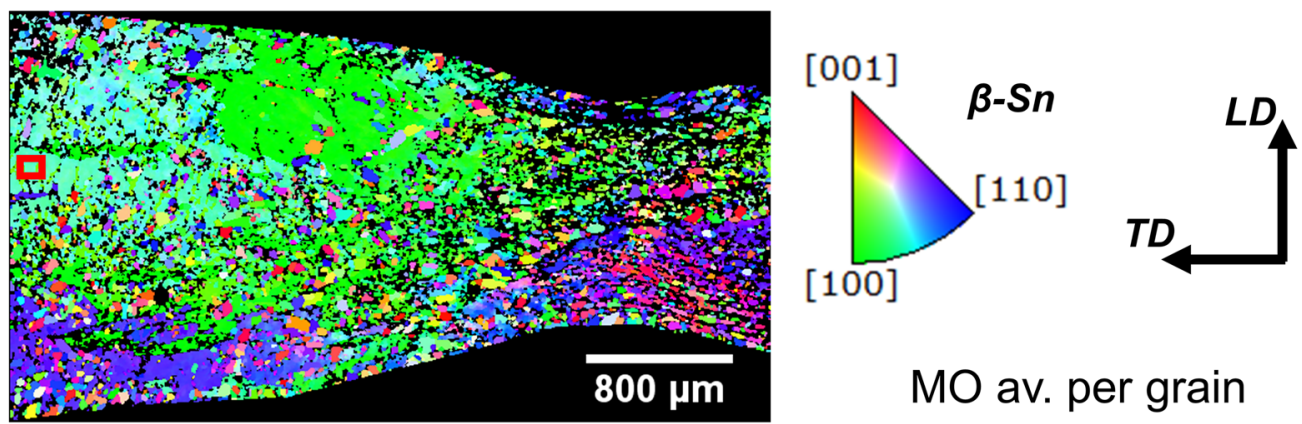

(b)

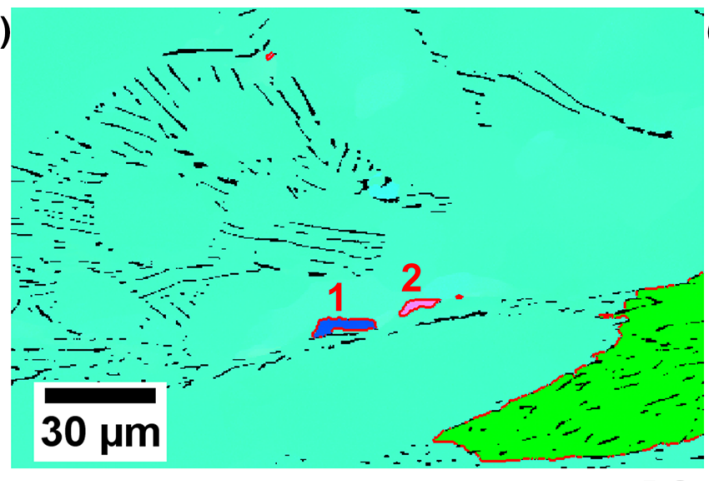

DS

(d)

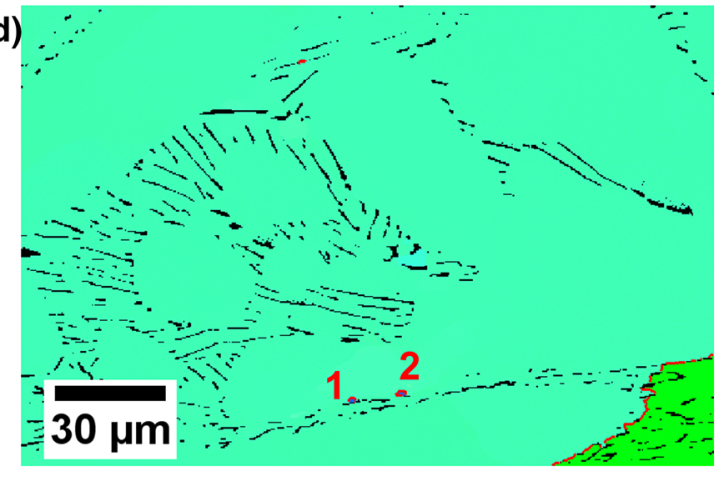

Onset secondary stage

(f)

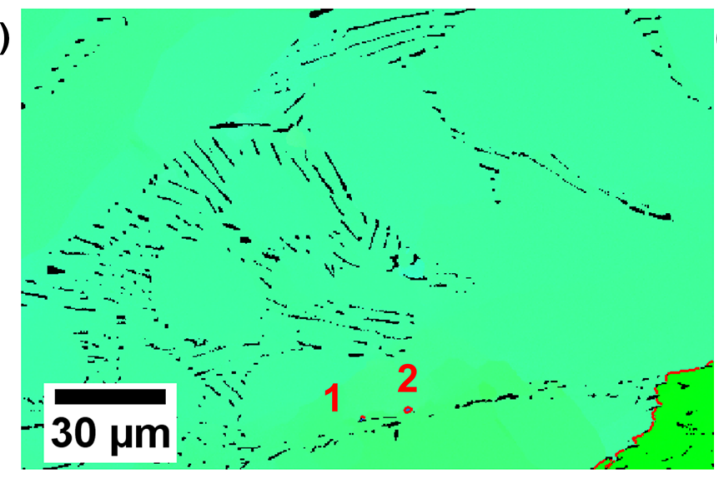

(e)
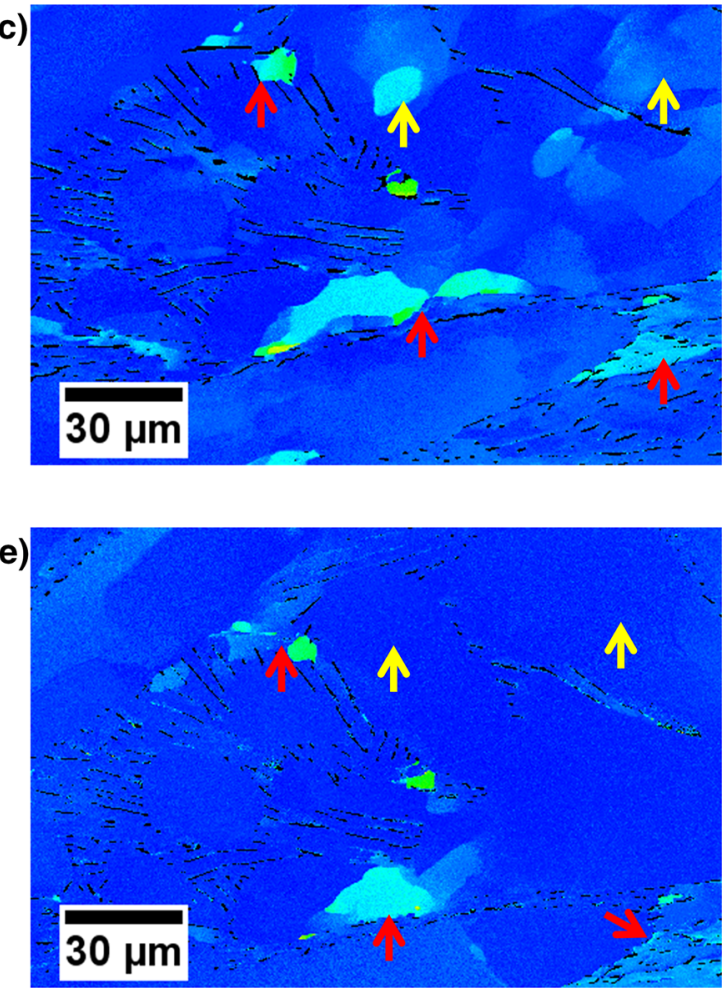

(g)

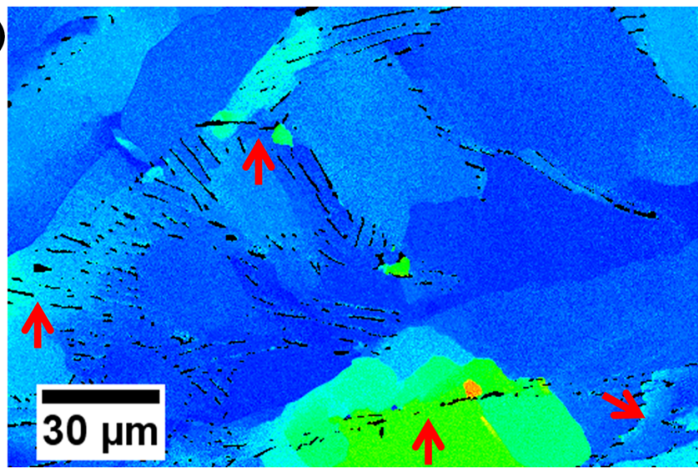

Tertiary stage

Fig. 4. EBSD crystal orientation mapping showing evolution of microstructural heterogeneity highlighting evolution within the uniformly deformed region for the bi-crystal sample. (a) The large scale IPF-LD map at tertiary stage creep. (b-g) Higher magnification IPF-LD maps of the red squared region in (a) within the non-necking region at different stages of creep (the grain boundaries larger than $5^{\circ}$ are labelled with red lines and the 2 embed small orientations are labelled with 1 and 2) (b, c) at the DS condition, (d, e) at the onset of secondary stage creep, and (f, $g)$ at the tertiary stage creep. With increasing strain, there is a gradual orientation change in the IPF-LD map (b, $d$, $f$ ), while the MO av. shows significant change, especially in eutectic regions near IMCs and at grain boundary (c, e, g). A common misorientation angle with respect to 'grain' as identified with EBSD thresholding of grain boundaries at $5^{\circ}$. 
(a)

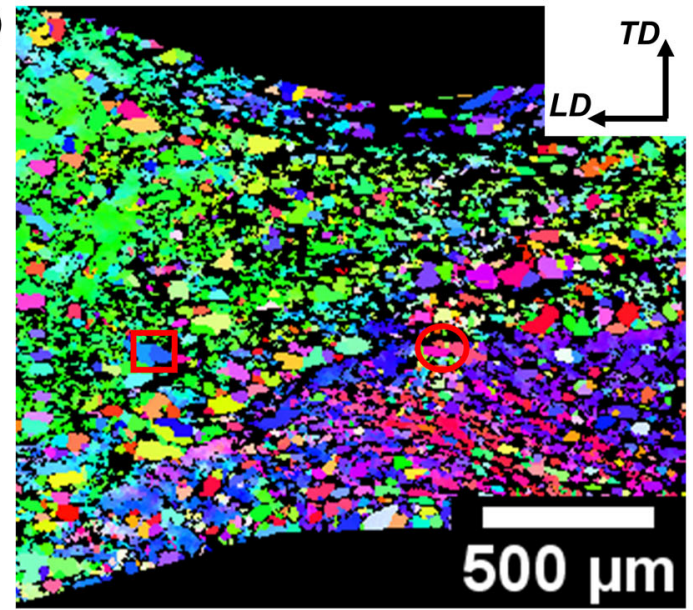

(c)

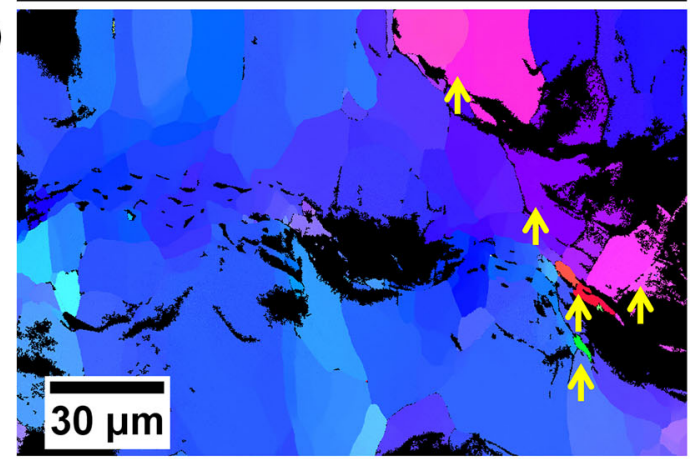

(e)

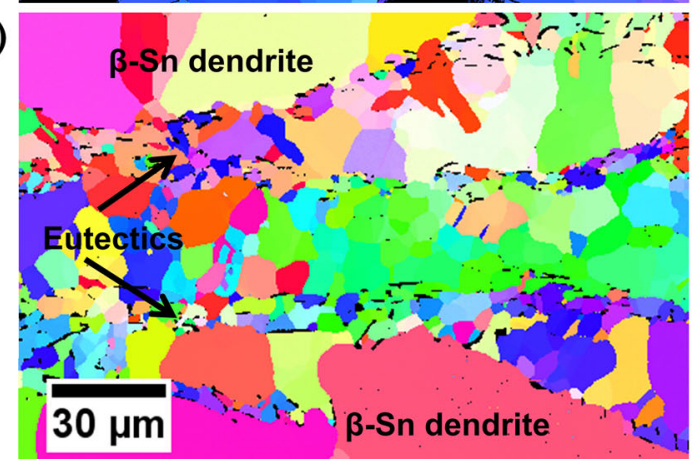

(b)

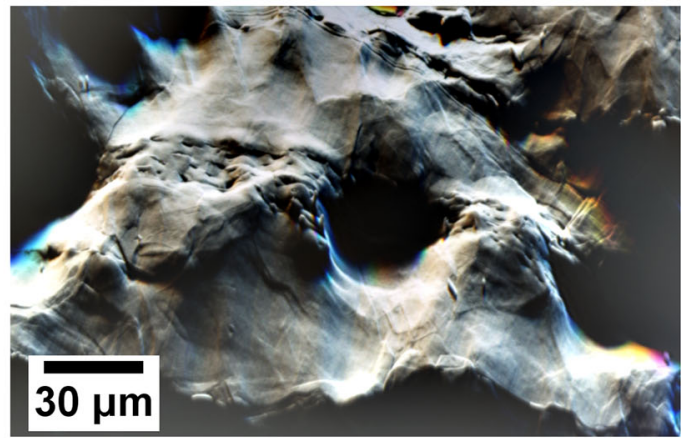

[001]

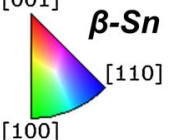

110] $\quad$ MO av. per grain

(d)

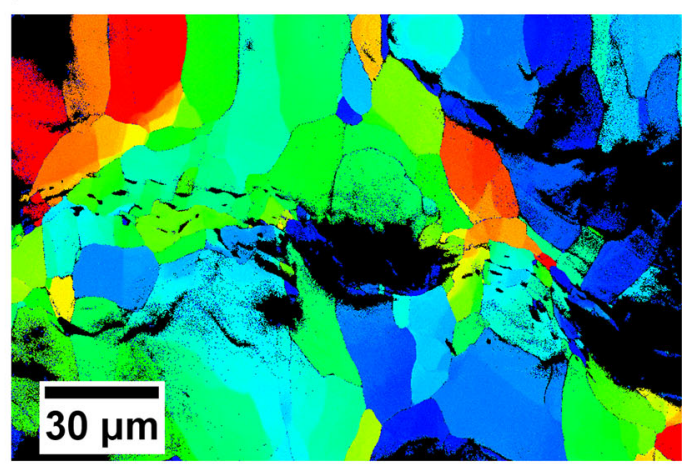

(f)

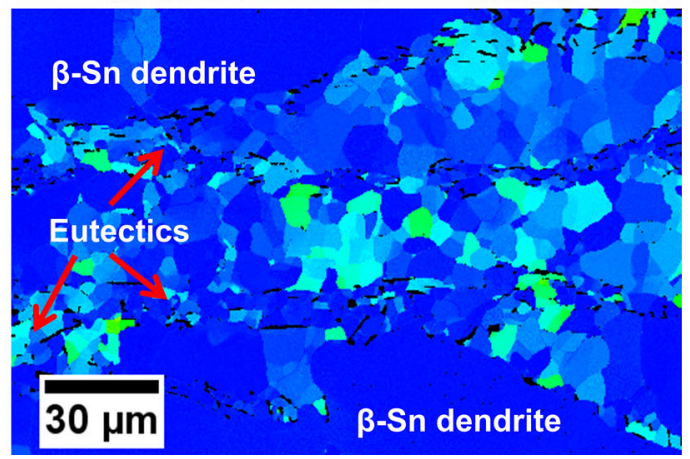

Fig. 5. EBSD crystal orientation mapping showing evolution of microstructural heterogeneity highlighting evolution within the necking region. (a) The large scale IPF-LD map for the bi-crystal sample of the necking region at tertiary stage creep. (b-f) Higher magnification IPF-LD maps of the same region in (a) within the necking region, $(b-d)$ in the red squared region, $(e, f)$ in the red circled region. With increasing strain, there is significant increase in surface roughness in the forescatter electron image (FSE) (b), increase in recrystallization volume in both dendrite and eutectic regions (the eutectic $\beta$-Sn has much smaller grain size than in dendrite) (c,e), and decrease in misorientation in the MO av. map (d, f).

eventually this leads to enough energy to drive recrystallization nuclei to segment the initial grain, which occurs in the tertiary stage creep.

With testing at higher temperatures the microstructure sensitivity between grains increases. A strong heterogeneous deformation is observed, even during the primary to secondary stage creep. There is even more heterogeneity associated in the secondary to tertiary stage creep.

\section{Strain Field and Crystal Orientation of [110] Single Crystal Sample}

A mainly single crystal orientation sample, which is between [100] and [110] in the loading direction was deformed at room temperature, i.e. $\sim 25^{\circ} \mathrm{C}$ $\left(\frac{T}{T_{\mathrm{M}}} \sim 0.6\right)$. The crystal unit cells of the orientations are shown with the main orientation highlighted by a red square together with the IPF-LD map (Fig. 6a).

From the strain field (Fig. $6 \mathrm{~b}$ and c), the sample deformed with a little heterogeneity at the starting point from the primary to secondary stage, but, in general, there is fairly homogenous deformation unlike the bi-crystal sample (Fig. 3). It reaches a strain level of $1.8 \%$ at the onset of secondary stage creep.

The DIC figures (Fig. 6d-f) show no obvious necking for this single crystal sample until fracture. 


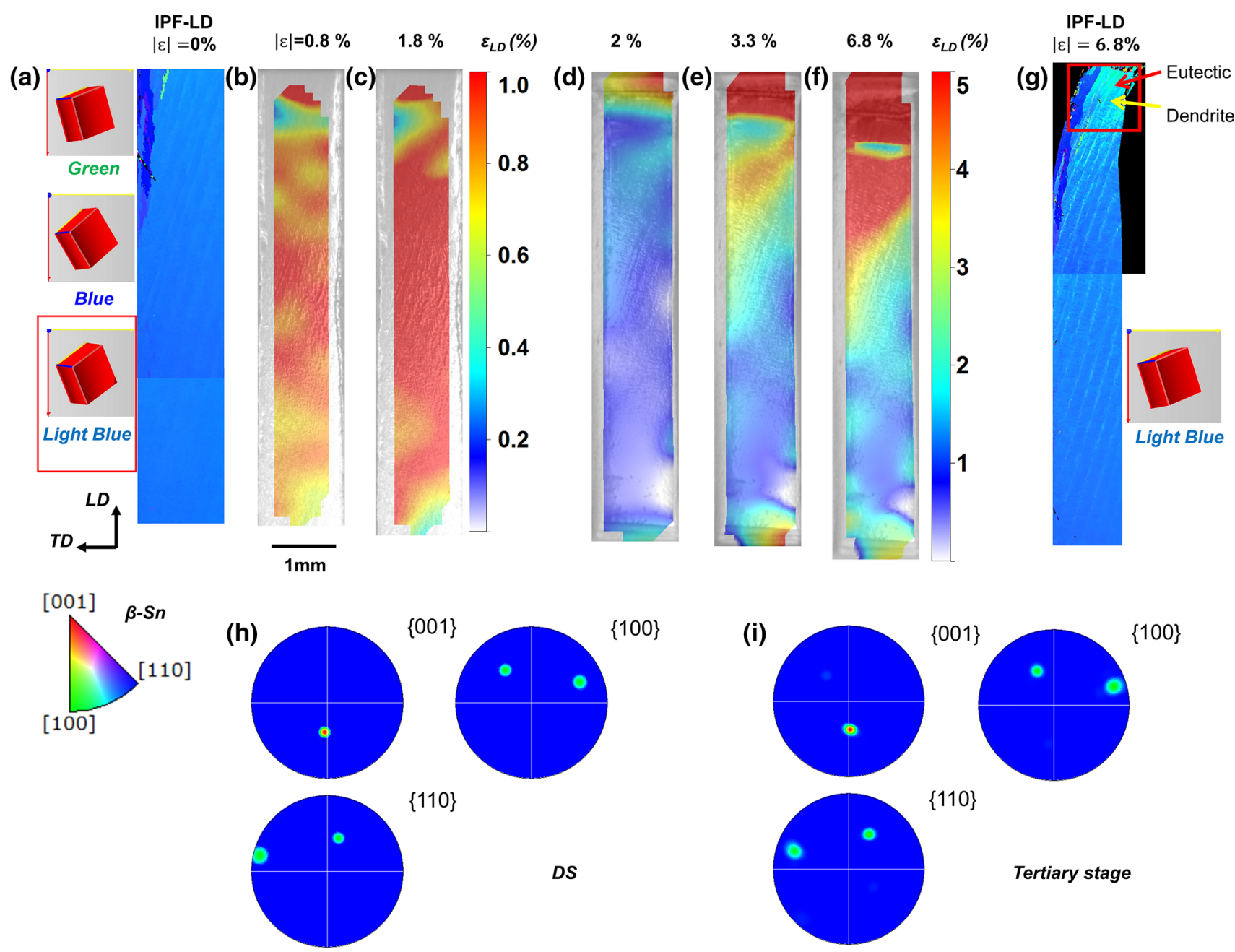

Fig. 6. The DIC figures and IPF-LD map of a single grain sample tested at room temperature. (a) The IPF-LD map after DS with crystal orientations shown (the main orientation highlighted with red square). (b, c) DIC figures interrupted near the onset of secondary stage creep at strain level $\left(\varepsilon_{\mathrm{L}}\right) 0.8 \%$ and $1.8 \%$ respectively. (d-f) DIC figures from secondary to tertiary stage creep (fracture) at strain level $2 \%, 3 \%$ and $6.8 \%$ respectively. $(\mathrm{g})$ The IPF-LD map at fracture $\left(\varepsilon_{\mathrm{LD}}=6.8 \%\right)$ with the crystal unit cell of the main orientation shown. (h, i) The PFs of the sample at DS condition (h), at tertiary stage creep (i). (Videos of the DIC fields are found in the Supplementary video S3 and S4).

This implies that this single-crystal is much more ductile than the bi-crystal sample (Fig. 3). From the IPF-LD map after fracture (Fig. 6g), the gauge of the sample is elongated (about $7 \%$ of its original length) and deformation is relatively stable in this creep regime. Within this deformed gauge, the dendrite arms are bent and thinned, likely due to deformation within tertiary stage creep. The DIC figures in Fig. 6d-f show strain from secondary to tertiary stage creep, which reveal the gradual increase in heterogeneity during deformation which is associated with the bending at the top end of the sample (Fig. 6g), where the dendrite and eutectic regions are indicated with red and yellow arrows.

The second crystal orientation, green, is appearing in the eutectic regions during creep, which is indicated with red arrows in Fig. 6g. The orientation changed smoothly by rotating towards [100]. The corresponding crystal unit cell shows slight rotation for the main orientation (Fig. 6g). A little spread in the $\mathrm{PF}$ is observed by comparing the asDS condition (Fig. $6 \mathrm{~h}$ ) with the one in tertiary stage creep (Fig. 6i). Recrystallization is only observed at the fracture surface and the detail of the microstructure is given in the next section (Figs. 7 and 8).

\section{Microstructure of the [110] Single-Crystal Sample Within the Non-necking Region}

The microstructure of the single-crystal sample within the non-necking region is shown in Fig. 7 for different stages of creep. It shows a clear single crystal orientation in the IPF-LD map (Fig. 7b and d) for both the DS condition and at the onset of secondary stage creep. The misorientation starts to develop in the eutectic region around IMCs (indicated with red arrows in Fig. 7e) at the onset of secondary stage creep, which is related to the 
(a)
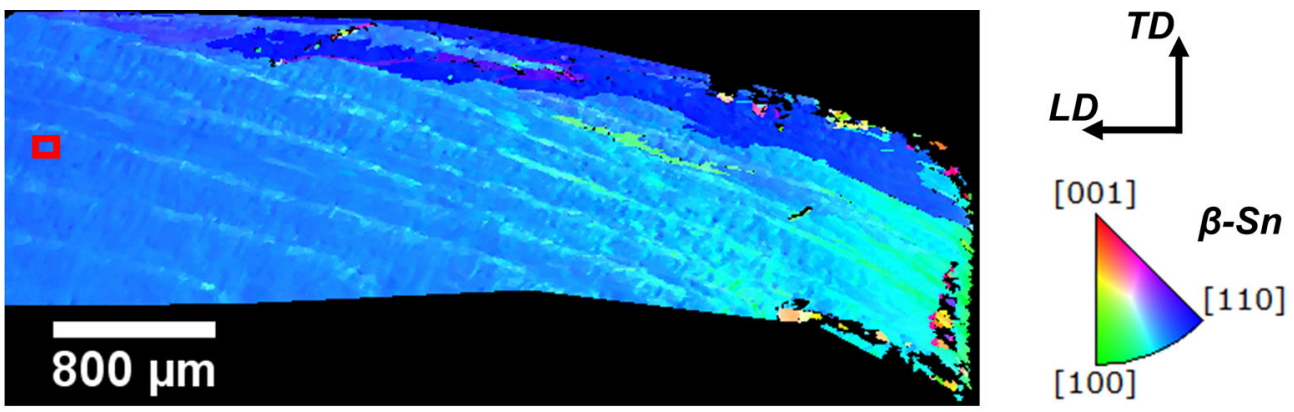

(b)

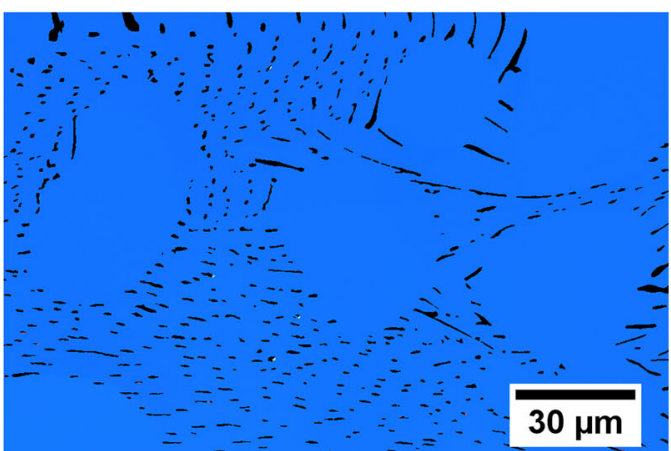

MO av. per grain

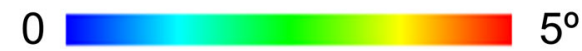

(c)

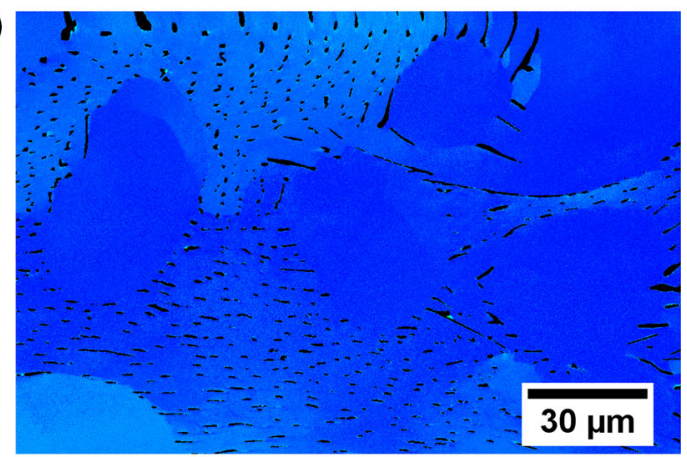

(d)

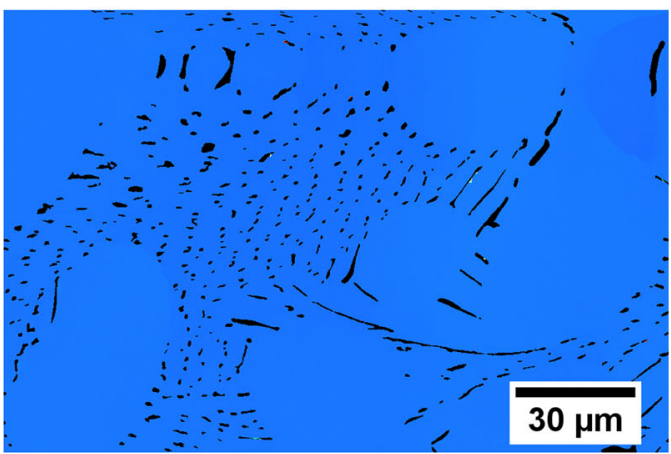

(e)

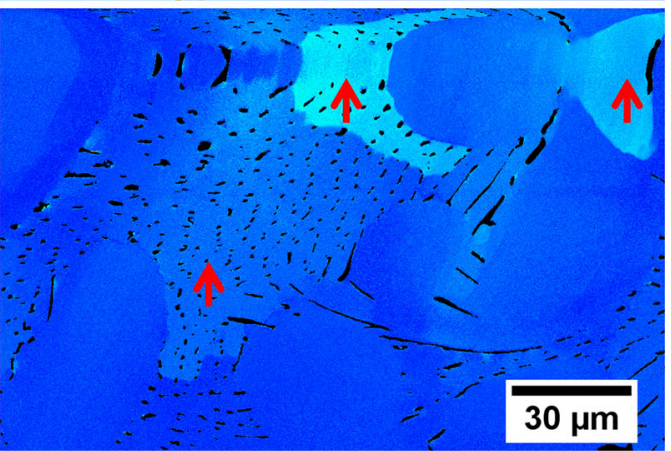

(f)

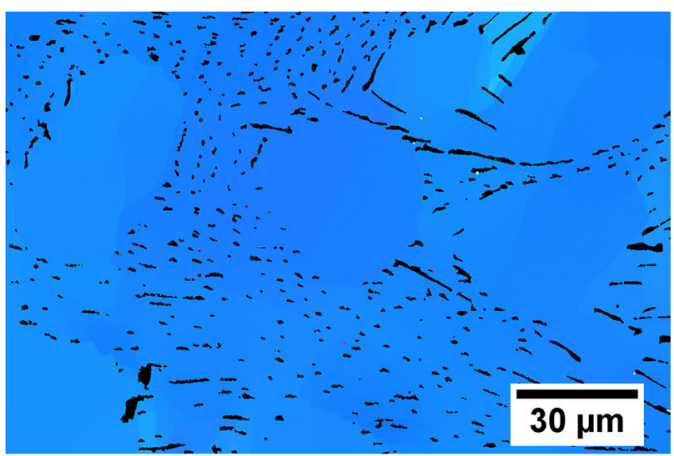

(g)

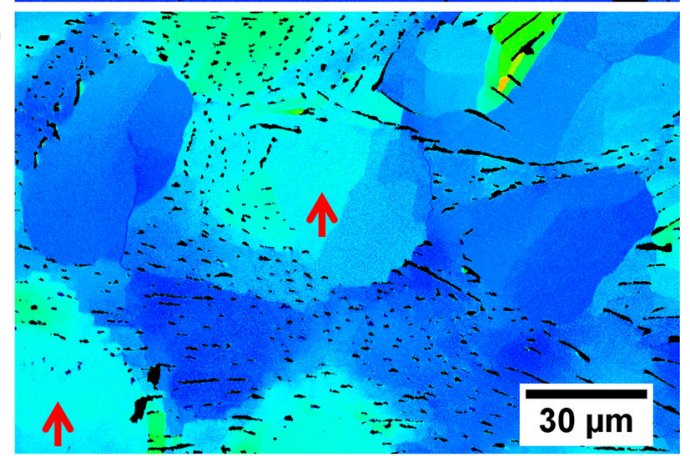

Tertiary stage

Fig. 7. EBSD crystal orientation mapping showing evolution of microstructural heterogeneity highlighting evolution within the uniformly deformed region of the single crystal sample. (a) The large scale IPF-LD map at fracture. (b-g) Higher magnification IPF-LD maps of the red squared region in (a) within the non-necking region at different stages of creep, (b, c) at the DS condition, (d, e) at the onset of secondary stage creep, and (f, g) at the tertiary stage creep. With increasing strain, there is slight change in the orientation in the IPF-LD map (b, $d, f)$ but the MO av. maps show significant increase, especially in eutectic regions (the MO hot spots are indicated with red arrows) (c, e, g) (Color figure online).

incoherent boundaries between IMCs and $\beta$-Sn. However, no special indication shows which IMCs, i.e., $\mathrm{Ag}_{3} \mathrm{Sn}$ and $\mathrm{Cu}_{6} \mathrm{Sn}_{5}$ localises more strain.
The misorientation in $\beta$-Sn dendrites has no obvious change from the primary to secondary stage creep, while the eutectic regions show increased 
(a)

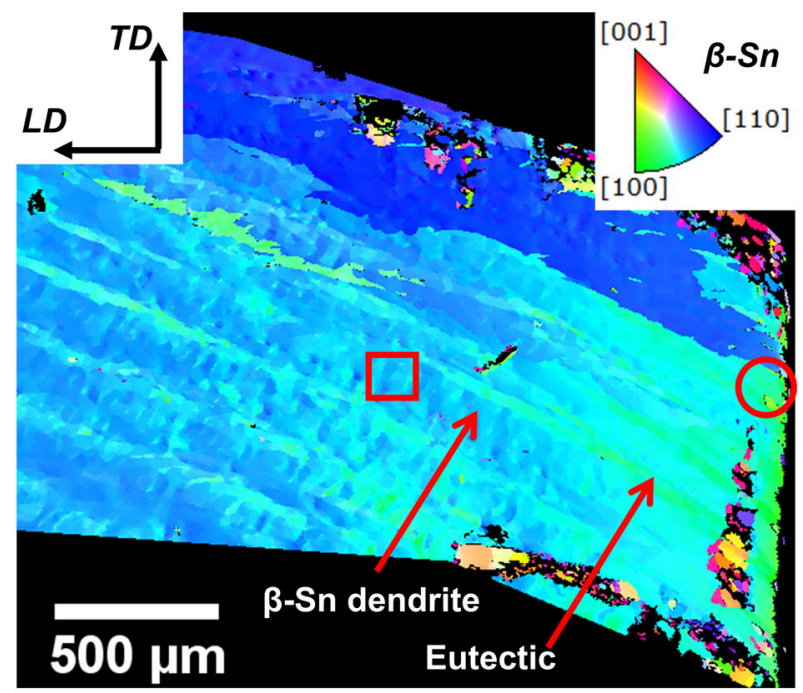

(c)

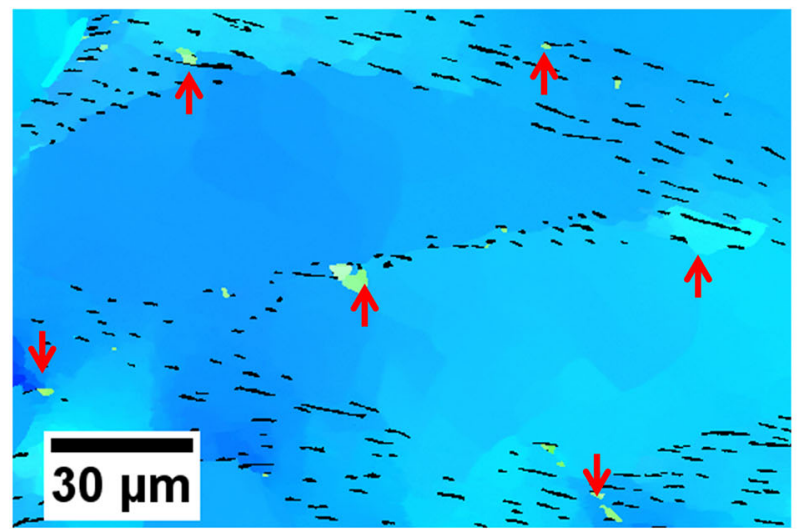

(e)

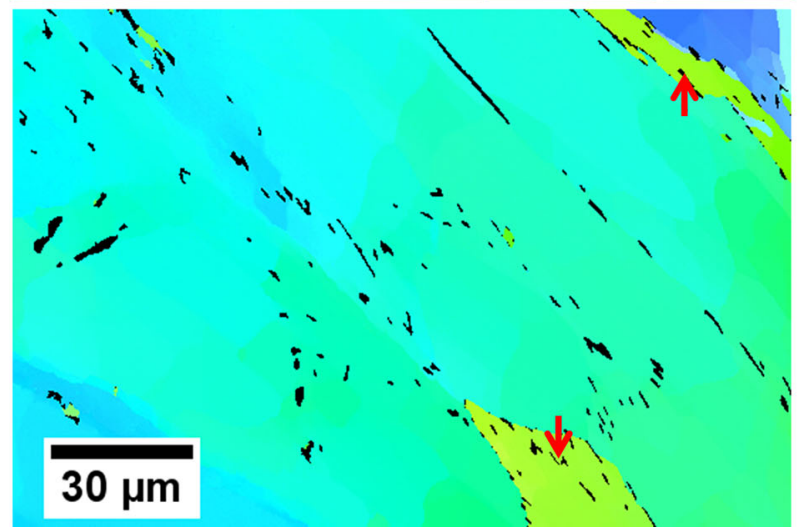

(b)

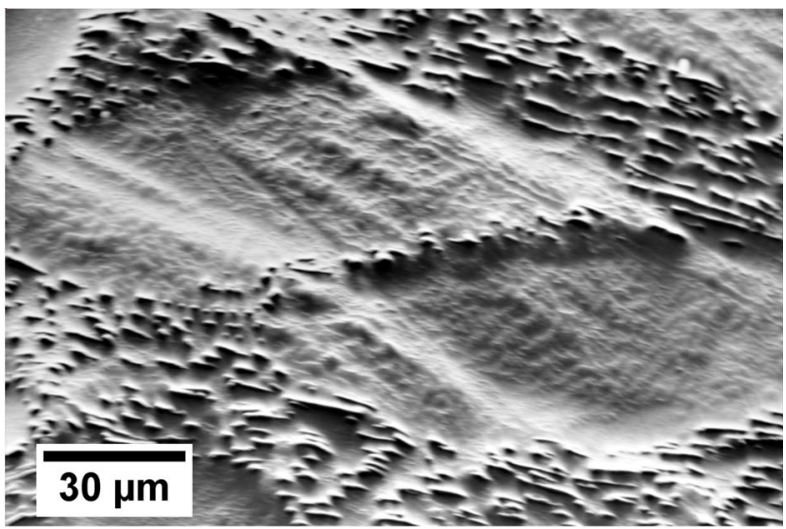

$\mathrm{MO}$ av. per grain

0

(d)

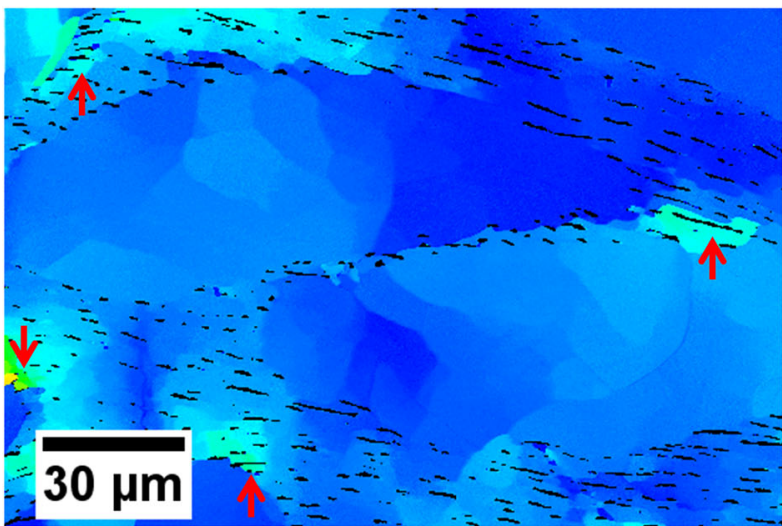

(f)

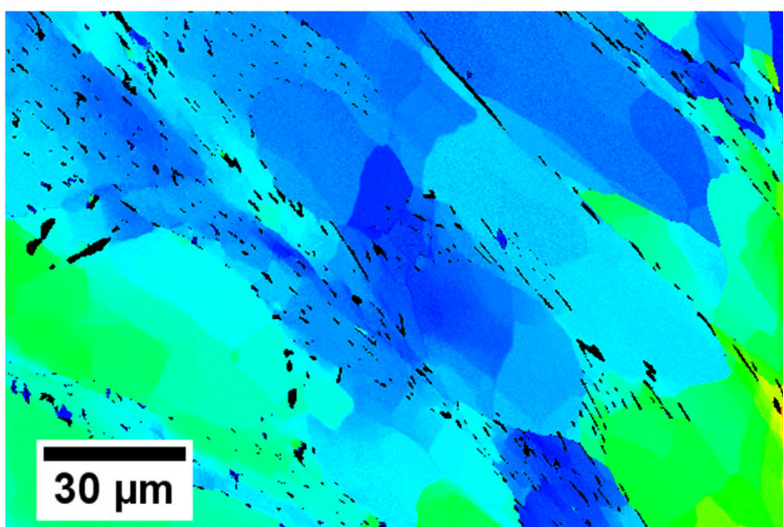

Fig. 8. EBSD crystal orientation mapping showing evolution of microstructural heterogeneity highlighting evolution within the bending neck. (a) The large scale IPF-LD map at fracture. (b-f) Higher magnification IPF-LD maps of the same region in (a) within the bending region, (b-d) in the red squared region, $(e, f)$ in the red circled region at the fracture surface. With increasing strain, there is significant increase in surface roughness in the forescatter electron image (FSE) (b), growth in recrystallized grain in the eutectic region (indicated with red arrows) (c, e), and increase in misorientation in the MO av. map (d, f) (Color figure online).

misorientation (Fig. 7c and e). A significant increase in misorientation is observed at the onset of the tertiary stage creep, and the high misorientation regions are mostly localised in the eutectic regions (Fig. 7g). The crystal orientation (Fig. 7f) is changed slightly by rotating towards the [100] direction and Fig. $7 \mathrm{~g}$ shows that misorientation starts to develop in the pure $\beta$-Sn regions and subgrains are formed. Furthermore, no obvious coarsening of IMCs is observed for the sample, and this particular region has not reached tertiary stage creep at the fracture point.

\section{Microstructure of the [110] Single-Crystal Sample Within the Necking Region}

The role of the IMCs on heterogeneous creep deformation is illustrated within a necked region of 
the single crystal sample deformed at room temperature, as shown in Fig. 8a. The undeformed crystal orientation of this single crystal has an orientation close to [110] (i.e., a darker blue) which is observed within the dendrite regions. The intermetallic-containing eutectic regions show significant $\beta$-Sn lattice rotation towards the [100] crystal direction (in green/cyan), indicating that there is more creep deformation within these microstructural regions, and this results in a clear demarcation of the underlying microstructure within the IPF map in Fig. 8a.

At the fracture surface, recrystallization developed (Fig. 8a). Within this field, the orientation field is also heavily modulated by the presence of the IMCs and the dendrite structure around them. The higher magnification IPF map within the bending region (Fig. 8b), shows that the $\beta$-Sn dendrites are elongated in the loading direction. The main crystal orientation is moving close to [100]. This implies that the initial crystal orientation is rotated gradually during creep to form the green-cyan orientation near [100] at the fracture surface (Fig. 8e). The second orientation (yellow, which is indicated with red arrows) is appearing in the eutectic regions near IMCs (Fig. 8c). Furthermore, it can be seen that the closer the distance to the fracture surface, the higher the misorientation of the sample (Fig. 8d and f).

Figure $8 d$ and $f$ show strong subgrain formation in both the $\beta$-Sn dendrite and eutectic regions. The eutectic $\beta$-Sn regions have more rotation relative to the $\beta$-Sn dendrites (Fig. 8e) and a new crystal orientation with a component towards [001] (yellow) develops more in the eutectic regions. The increase in surface roughness is less notable as in the bicrystal sample (Fig. 8c), and this is likely due to the lack of recrystallization and more homogenous deformation processes. Also, the MO av. illustrated in Fig. $8 \mathrm{f}$ has a much higher value than in the bicrystal sample (Fig. 5f) at the fracture tip, which indicates that there is significant stored energy within this region.

\section{CONCLUSIONS}

The creep behaviour and microstructural evolution of bulk SAC305 solder alloy are studied in this project. Controlled microstructures were generated by solidifying dog-bone samples by Bridgman growth which produced a similar number of $\beta$-Sn grains, $\beta$-Sn orientations, and lengthscale of the eutectic $\mathrm{Ag}_{3} \mathrm{Sn}$ and $\mathrm{Cu}_{6} \mathrm{Sn}_{5}$ in all samples prior to creep testing.

The samples tested here have key similarities to the microstructure of a real SAC solder joint, i.e., a similar number of tin grains (1-3), the similar microstructures with $\beta$-Sn dendrites, and fine IMCs in eutectic regions (but the IMCs here are larger than found in a typical joint to aid characterisation here). The Bridgman grown samples enable consistent microstructures to be tested and promote understanding of fundamental mechanisms. This avoids addressing the stochastic nature of real solder joint microstructures, which have variations from joint to joint due to differences in undercooling. A benefit of using larger IMCs is to enhance the localisation of strain during creep deformation, so as to make it easier to observe the difference between the phases (the nature of heterogeneous deformation) during creep. Further work is planned to explore the role of IMCs size on strain localisation during deformation.

The following conclusion can be drawn from this work.

- At a constant stress of $30 \mathrm{MPa}$, shorter secondary creep lifetime and greater secondary creep strain rate are obtained at elevated temperature. The strain at the onset of secondary creep decreases with temperature, which is caused by less dislocation formation and motion, but more diffusion at higher temperatures, i.e., recovery takes place at lower creep strain level at higher temperature.

- A significant change in secondary creep strain rate is found beyond homogenous temperature $\sim \frac{T}{T_{\mathrm{M}}}=0.7$. This is associated with a change in creep mechanism from climb-controlled dislocation creep to lattice diffusion creep. This causes a decrease in the activation energy, $Q$, in the high temperature regime (363-473 K) relative to the low temperature regime (298$333 \mathrm{~K}$ ), the values of $Q$ are $41 \mathrm{~kJ} / \mathrm{mol}$ and $6 \mathrm{~kJ} / \mathrm{mol}$, respectively.

- Ductile failure was observed for all single, bicrystal and tri-crystal samples in tertiary stage creep. Bi-crystal and tri-crystal samples tend to deform with stronger heterogeneity and less ductility than single-crystal samples (see supplementary Figures S1-S4). This deformation heterogeneity is associated with the presence of the grain boundary. Moreover, the [100] crystal orientation seems to be able to carry more creep strain and deform with less ductility than the [110] crystal orientation during creep.

- The deformation of the SAC305 samples is initiated in the eutectic regions near IMCs with high misorientation development and runs through the $\beta$-Sn dendrite by grain rotation. When rotated enough, this leads to subgrain formation, and provides sufficient energy to generate recrystallization which is localised near the ultimate fracture point of each specimen. This failure phenomenon is enhanced at elevated temperature due to the increase in dislocation climb and grain boundary mobility.

DS SAC305 contains two distinct microstructural regions, containing primary $\beta$-Sn dendrites and a eutectic consisting of $\beta$-Sn and fine scale IMCs. 
These deform differently during creep. An increase in $\mathrm{MO}$ av. is observed in regions close to the IMCs which highlights the role of strain gradients in these regions of high microstructure contrast. These regions seem to dominate recrystallization events, perhaps due to the role of stored energy which can act to initiate recrystallization. Deformation within the eutectic and around the IMCs is highly localised, whereas the deformation within the dendrite is more spatially extensive. The difference in deformation patterning results in a change in the localisation of stored energy and therefore the likelihood of local recrystallization. 'Rainbow' recrystallization is promoted in the later stages of creep, where grains with a multiplicity of orientations are generated in the single crystal regions. The length scale of this recrystallization is related to the dendrite and IMCs spacing for each region.

\section{ACKNOWLEDGMENTS}

TBB would like to thank the Royal Academy of Engineering for his research fellowship. CG would like to thank EPSRC (EP/M002241/11) for funding of his research fellowship. We would like to thank Nihon Superior for positive support and encouragement to conduct this work. We thank Dr. Sergey Belyakov and Ning Hou for support in the initial fabrication of the samples. The assistance of TeCheng $\mathrm{Su}$ in DIC studies is also gratefully acknowledged. We acknowledge the preliminary work of Grey Chen that supported the initial development of this project. The microscope and loading frame used to conduct these experiments was supported through funding from Shell Global Solutions and is provided as part of the Harvey Flower EM suite at Imperial.

\section{AUTHOR CONTRIBUTIONS}

TG drafted the initial manuscript and conducted the experimental work. CG and TBB supervised the work equally. All authors contributed to the final manuscript.

\section{ELECTRONIC SUPPLEMENTARY MATERIAL}

The online version of this article (https://doi.org/ 10.1007/s11664-018-6744-1) contains supplementary material, which is available to authorized users.

\section{OPEN ACCESS}

This article is distributed under the terms of the Creative Commons Attribution 4.0 International License (http://creativecommons.org/licenses/by/4.0/), which permits unrestricted use, distribution, and reproduction in any medium, provided you give appropriate credit to the original author(s) and the source, provide a link to the Creative Commons license, and indicate if changes were made.

\section{REFERENCES}

1. H. Black, Environ. Health Perspect. 113, 683 (2005).

2. R.J. Coyle, K. Sweatman, and B. Arfaei, JOM J. Miner. Met. Mater. Soc 67, 2394 (2015).

3. W. Zhu, L. Xu, J.H. Pang, X. Zhang, E. Poh, Y. Sun, A.Y. Sun, C. Wang, and H. Tan, 58th Electronic Components and Technology Conference, 2008. ECTC 2008 (2008).

4. E.H. Wong, S. Seah, W. Van Driel, N. Owens, and Y.S. Lai, Microelectron. Reliab. 49, 139 (2009).

5. T.R. Bieler, H. Jiang, L.P. Lehman, T. Kirkpatrick, E.J. Cotts, and B. Nandagopal, IEEE Trans. Compon. Packag. Technol. 31(2), 370 (2008).

6. T.R. Bieler, B. Zhou, L. Blair, A. Zamiri, P. Darbandi, F. Pourboghrat, T.K. Lee, and K.C. Liu, J. Electron. Mater. 41(2), 283 (2012).

7. K.W. Moon, W.J. Boettinger, U.R. Kattner, F.S. Biancaniello, and C. Handwerker, J. Electron. Mater. 29(10), $1122(2000)$.

8. C.K. Lin and D.Y. Chu, J. Mater. Sci. Mater. Electron. 16(6), 355 (2005).

9. N. Hiyoshi, Mech. Eng. J. 3(3), 16-00081 (2016).

10. Y. Kariya, T. Asai, and T. Suga, in Third International Conference on Experimental Mechanics and Third Conference of the Asian Committee on Experimental Mechanics (2005).

11. M. Mathew, H. Yang, S. Movva, and K. Murty, Metall. Mater. Trans. A. 36(1), 99 (2005).

12. T.W. Woo, M. Sakane, K. Kobayashi, H.C. Park, and K.-S. Kim, J. Microelectron. Packag. Soc. 17(3), 33 (2010).

13. R. Sidhu and N. Chawla, Metallurgical and Materials Transactions A. 39(2), 340 (2008).

14. R. Sidhu, X. Deng, and N. Chawla, Metall. Mater. Trans. A. 39(2), 349 (2008).

15. J.H. Pang, B. Xiong, and T. Low, in Proceedings of the 54th Electronic Components and Technology Conference, 2004 (2004).

16. P.T. Vianco, J.A. Rejent, and A.C. Kilgo, J. Electron. Mater 33(11), 1389 (2004).

17. P.T. Vianco, J.A. Rejent, and A.C. Kilgo, J. Electron. Mater 33(12), 1473 (2004).

18. S. Choi, J. Lee, F. Guo, T. Bieler, K. Subramanian, and J. Lucas, JOM J. Miner. Met. Mater. Soc. 53(6), 22 (2001).

19. A. Fahim, S. Ahmed, M.R. Chowdhury, J.C. Suhling, and P. Lall, in 2016 15th IEEE Intersociety Conference on Thermal and Thermomechanical Phenomena in Electronic Systems (ITherm) (2016).

20. M. Hasnine, J. Suhling, B. Prorok, M. Bozack, and P. Lall, Exp. Mech. 57(4), 603 (2017).

21. D. Herkommer, M. Reid, and J. Punch, J. Electron. Mater. 38(10), 2085 (2009).

22. K.O. Lee, J.W. Morris, and F. Hua, J. Electron. Mater. 42(3), 516 (2013)

23. L. Xu, W.B. Tang, Z.H. Zhu, Y.P. Wu, L.F. Ren, and Y. Yao, DEStech Trans. Eng. Technol. Res. (amme). (2017).

24. S. Mukherjee, P. Chauhan, M. Osterman, A. Dasgupta, and M. Pecht, J. Electron. Mater. 45(7), 3712 (2016).

25. T. Ogawa, R. Kaga, and T. Ohsawa, J. Electron. Mater. 34(3), 311 (2005)

26. Q.K. Zhang and Z.F. Zhang, Acta Materialia. 59(15), 6017 (2011).

27. Q.K. Zhang and Z.F. Zhang, Scripta Materialia. 67(3), 289 (2012).

28. B. Talebanpour, U. Sahaym, and I. Dutta, IEEE Trans. Dev. Mater. Reliab. 16(3), 318 (2016).

29. A.A. El-Daly, A.M. El-Taher, and T.R. Dalloul, J. Alloys Compd. 587, 32 (2014).

30. X. Chen, J. Zhou, F. Xue, and Y. Yao, Mater. Sci. Eng. A 662, 251 (2016).

31. C.R. Mayer, S. Lotfian, J. Molina-Aldareguia, and N. Chawla, Adv. Eng. Mater. 17(8), 1168 (2015). 
32. J. Han, J. Sun, T. Wen, and F. Guo, J. Mater. Sci. Mater. Electron. 1 (2018).

33. T.R. Bieler and A.U. Telang, J. Electron. Mater. 38(12), 2694 (2009).

34. B. Arfaei, S. Mahin-Shirazi, S. Joshi, M. Anselm, P. Borgesen, E. Cotts, J. Wilcox, and R. Coyle, in 2013 IEEE 63rd Electronic Components and Technology Conference (ECTC) (2013).

35. J. Han, S. Tan, and F. Guo, J. Electron. Mater. 45(12), 6086 (2016).

36. S. Tan, J. Han, and F. Guo, J. Electron. Mater. 47(7), 4156 (2018).

37. Y. Zuo, T.R. Bieler, Q. Zhou, L. Ma, and F. Guo, J. Electron. Mater. 47(3), 1881 (2018)

38. I. Anderson, B. Cook, J. Harringa, and R. Terpstra, JOM J. Miner. Met. Mater. Soc. 54(6), 26 (2002).

39. B. Arfaei, N. Kim, and E. Cotts, J. Electron. Mater. 41(2), 362 (2012).

40. C. Gourlay, S. Belyakov, Z. Ma, and J. Xian, JOM J. Miner. Met. Mater. Soc. 67(10), 2383 (2015).
41. T.K. Lee, B. Zhou, L. Blair, K.C. Liu, and T.R. Bieler, J. Electron. Mater. 39(12), 2588 (2010).

42. Z. Ma, S. Belyakov, K. Sweatman, T. Nishimura, and C. Gourlay, Nat. Commun. 8(1), 1916 (2017)

43. L.P. Lehman, Y. Xing, T.R. Bieler, and E.J. Cotts, Acta Materialia. 58(10), 3546 (2010).

44. J. Xian, Z. Ma, S. Belyakov, M. Ollivier, and C. Gourlay, Acta Mater. 123, 404 (2017).

45. S. Yang, Y. Tian, and C. Wang, J. Mater. Sci. Mater. Electron. 21(11), 1174 (2010).

46. J. Han and F. Guo, J. Mater. Sci. Mater. Electron. 1 (2018)

47. R.J. Schaefer and D.J. Lewis, Metall. Mater. Trans. A. 36(10), 2775 (2005).

48. P.D. Pereira, J.E. Spinelli, and A. Garcia, Mater. Des. 45, 377 (2013).

49. N. Hou, S. Belyakov, L. Pay, A. Sugiyama, H. Yasuda, and C. Gourlay, Acta Mater. 149, 119 (2018).

50. J. Jiang, T. Zhang, F.P. Dunne, and T.B. Britton, Proc. $R$. Soc. A. 472(2185), 20150690 (2016). 